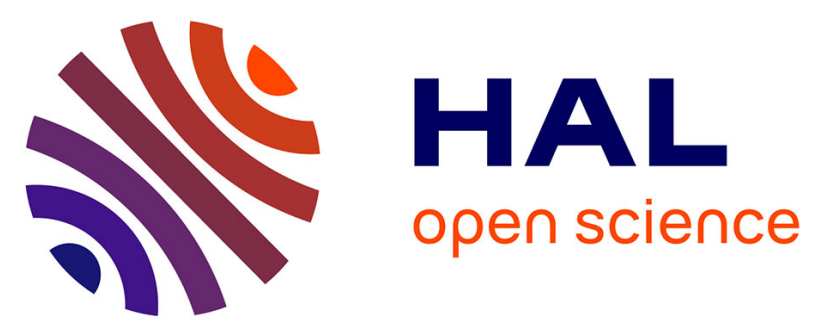

\title{
Representativeness of airborne brake wear emission for the automotive industry: A review
}

Florian Philippe, Martin Morgeneyer, Maiqi Xiang, Maheandar Manokaran,

Brice Berthelot, Yan-Ming Chen, Pierre Charles, Frédéric Guingand, Christophe Bressot

\section{To cite this version:}

Florian Philippe, Martin Morgeneyer, Maiqi Xiang, Maheandar Manokaran, Brice Berthelot, et al.. Representativeness of airborne brake wear emission for the automotive industry: A review. Proceedings of the Institution of Mechanical Engineers, Part D: Journal of Automobile Engineering, 2021, 235 (10-11), pp.2651-2666. 10.1177/0954407021993011 . ineris-03340278

\section{HAL Id: ineris-03340278}

https://hal-ineris.archives-ouvertes.fr/ineris-03340278

Submitted on 19 Oct 2021

HAL is a multi-disciplinary open access archive for the deposit and dissemination of scientific research documents, whether they are published or not. The documents may come from teaching and research institutions in France or abroad, or from public or private research centers.
L'archive ouverte pluridisciplinaire HAL, est destinée au dépôt et à la diffusion de documents scientifiques de niveau recherche, publiés ou non, émanant des établissements d'enseignement et de recherche français ou étrangers, des laboratoires publics ou privés. 


\section{Representativeness of airborne brake wear emission for the automotive industry: A review}

Florian PHILIPPE ${ }^{1,2}$, Martin MORGENEYER ${ }^{1}$, Maiqi XIANG ${ }^{1}$, Maheandar MANOKARAN ${ }^{1}$, Brice BERTHELOT ${ }^{4}$, Yan-ming CHEN $^{3}$, Pierre CHARLES ${ }^{2}$, Frédéric GUINGAND ${ }^{2}$, Christophe BRESSOT4

${ }^{1}$ University of Technology of Compiègne, Centre Pierre Guillaumat, 60200 Compiègne , France, maiqi.xiang@utc.fr; martin.morgeneyer@utc.fr

${ }^{2}$ Groupe PSA, Route de Gisy, 78140 Vélizy-Villacoublay, France florian.philippe@mpsa.com; pierre.charles@mpsa.com; frederic.guigand@mpsa.com

${ }^{3}$ CETIM, 52 Avenue Félix Louat, 60300 Senlis, France, yanming.chen@cetim.fr

${ }^{4}$ INERIS, Rue Jacques Taffanel, 60550 Verneuil-en-Halatte, France, christophe.bressot@ineris.fr

Corresponding Author: Florian PHILIPPE: florian.philippe@utc.fr

\section{Abstract}

Brake wear gives $16 \%$ to $55 \%$ by mass to total non-exhaust traffic related PM10 emissions in urban environments. While engines have become cleaner in the past decades, few improvements were made to lower non-exhaust emission until recently. Researchers have developed several experimental methods over the past years to assess brake emissions. However, observations tend to differ from a method to another with respect to many disciplines, ranging from particle system characterization to brake cycles, and it remains difficult to compare results of different research groups. It is so crucial to get a consensus on the standard experimental method. The following article lists limits which influence measurements and has to be taken into account when comparing works from different laboratories. This article also discusses how to design tests to get a relevant braking particle system characterization.

Keywords: brake emission, representativeness, non-exhaust emission, dynamometer bench, pin-on-disc, sampling system 


\section{Index}

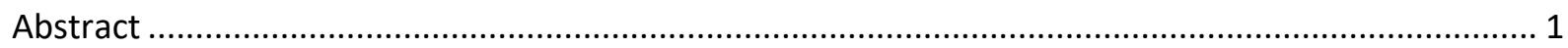

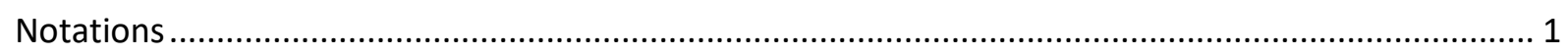

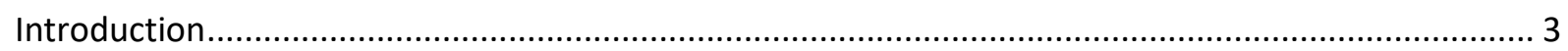

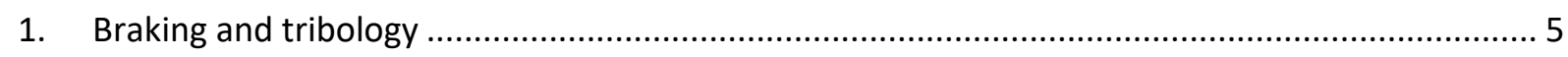

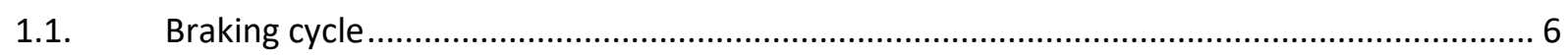

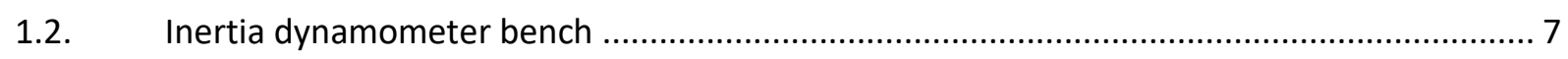

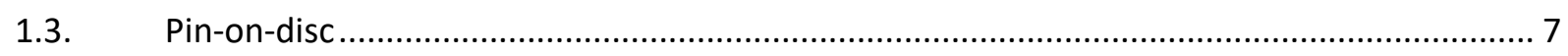

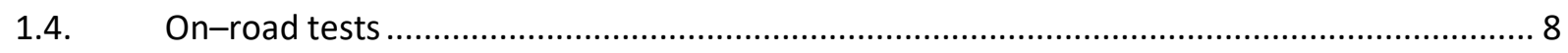

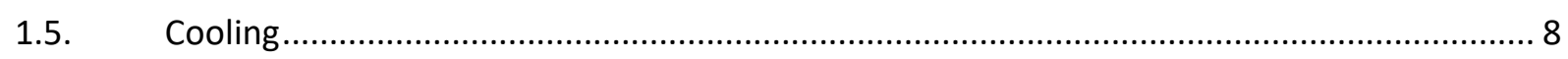

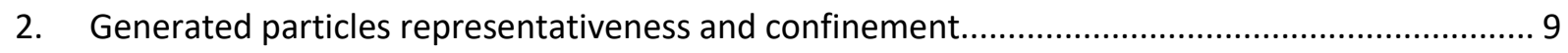

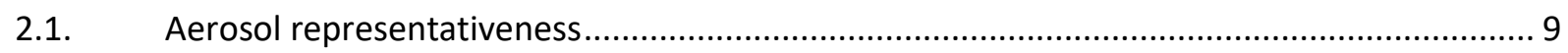

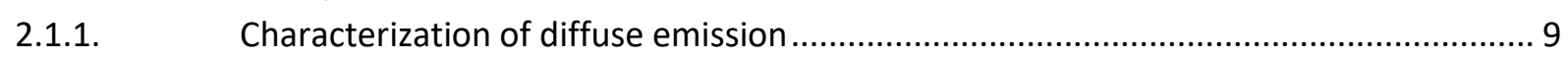

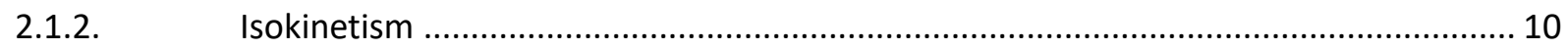

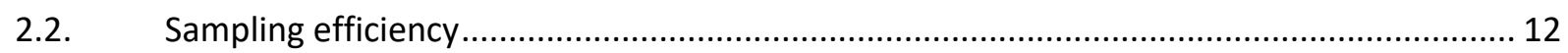

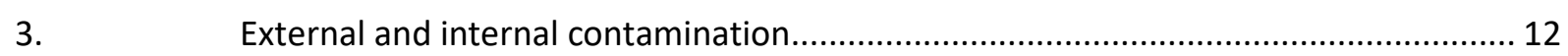

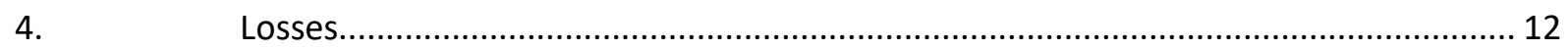

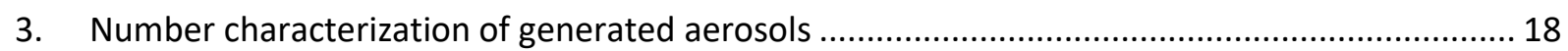

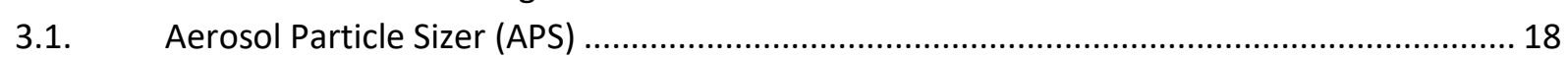

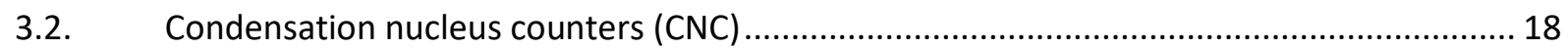

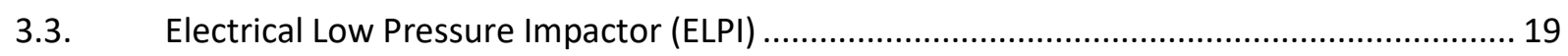

3.4. Scanning Mobility Particle sizer Spectrometer (SMPS) .................................................... 19

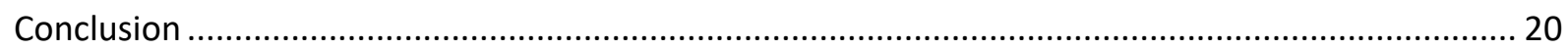

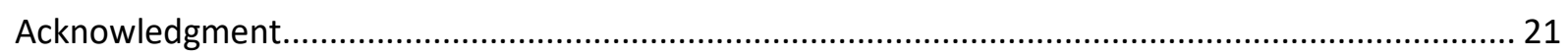

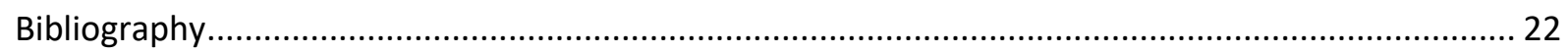

\section{Notations}

APS : Aerodynamic Particle Sizer

$C_{c}$ : Slip correction factor (no unit)

D: Diffusion coefficient $\left(\mathrm{m}^{2} / \mathrm{s}\right)$

$d_{p}$ : Aerodynamic diameter of the particle $(\mathrm{m})$

DustTrak: DustTrak aerosol monitor

ELPI: Electrical Low-Pressure Impactor

FMPS: Fast Mobility Particle Sizer Spectrometer

GRIMM: GRIMM optical aerosol spectrometer

$\mathrm{h}$ : Chamber or pipe height $(\mathrm{m})$

$\mathrm{k}$ : Boltzmann constant $\left(=1,38 \cdot 10^{-23} \mathrm{~J} \cdot \mathrm{K}^{-1}\left(\right.\right.$ or $\left.\left.\mathrm{kg} \cdot \mathrm{m}^{2} \cdot \mathrm{s}^{-2} \cdot \mathrm{K}^{-1}\right)\right)$

$\mathrm{L}$ : Characteristic linear dimension of the duct $(\mathrm{m})$

$l_{0}$ : Characteristic dimension of the conduct $(\mathrm{m})$

LSA: Laser Scattering Analyzer

OPS: Optical Particle Sizer

Q: Volumetric fluid flow $\left(\mathrm{m}^{3} \cdot \mathrm{s}^{-1}\right)$

$Q_{\text {total: }}$ Total inlet or outlet flow $\left(\mathrm{m}^{3} \cdot \mathrm{min}^{-1}\right)$ 
Re: Reynolds number (no unit)

Sh: Sherwood number (no unit)

SMPS: Scanning Mobility Particle sizer Spectrometer

Stk: Stokes number (no unit)

$\mathrm{T}$ : Temperature $(\mathrm{K})$

$\mathrm{U}$ : Fluid velocity $\left(\mathrm{m} . \mathrm{s}^{-1}\right)$

$\mathrm{U}_{\mathrm{t}}$ : Turbulent inertial deposition velocity $\left(\mathrm{m} . \mathrm{s}^{-1}\right)$

$\mathrm{U}_{+}$: Dimensionless deposition velocity (no unit)

$\mathrm{U}_{\text {inlet }}$ : Sampling velocity $\left(\mathrm{m} . \mathrm{s}^{-1}\right)$

$\mathrm{U}_{\text {local }}$ : Velocity of the fluid in the duct $\left(\mathrm{m}_{\mathrm{s}} \mathrm{s}^{-1}\right)$

$U_{\text {set: }}$ : Terminal settling velocity $\left(\mathrm{m}^{\left.-\mathrm{s}^{-1}\right)}\right.$

$V_{\text {chamber: }}$ Volume of the chamber $\left(\mathrm{m}^{3}\right)$

WLTP: Worldwide harmonized Light vehicles Test Procedure

Z: gravitional settling parameter (no unit)

$\beta_{\text {set }}$ : Losses due to settling (no unit)

$\eta_{\text {bend }}$ : Transport efficiency through a bend (no unit)

$\eta_{t u r b}$ : Transport efficiency with turbulence losses

$\eta_{\text {tube,diff }}$ : Transport efficiency with diffusion losses (no unit)

$\varnothing$ : Pipe section $(\mathrm{m})$

$\varnothing_{\text {inlet: }}$ Diameter of the inlet section $(\mathrm{m})$

$\varnothing_{\text {local }}$ : Diameter of the duct where the air is sampled $(\mathrm{m})$

$\lambda$ : Mean free path length of the gas molecules $(\mathrm{m})$

$\mu_{g}$ : Fluid dynamic viscosity $\left(\mathrm{kg} \cdot \mathrm{m}^{-1} \cdot \mathrm{s}^{-1}\right)$

$\rho:$ Fluid volumetric mass $\left(\mathrm{kg} \cdot \mathrm{m}^{-3}\right)$

$\rho_{\mathrm{p}}$ : Volumetric mass of the particle $\left(\mathrm{kg} \cdot \mathrm{m}^{-3}\right)$

$\tau_{0}$ : Fluid shear stress $\left(\mathrm{kg} \cdot \mathrm{m}^{-1} \cdot \mathrm{s}^{-2}\right)$

$\tau_{\text {chamber }}$ : Complete renewal chamber air $\left(\mathrm{min}^{-1}\right)$

$\tau_{\text {set }}$ : Duration of dropping of a particle in chamber or pipe (s)

\section{Introduction}

The Cambridge dictionary defines a brake as "a device that makes a vehicle go slower or stop, or a pedal, bar, or handle that makes this device work". In the automobile field, three options are possible to slow down a vehicle such as electromagnetic system, hydraulic system, or frictional brake system. Because of its high market share, the discussed type here is only the frictional brake, and more specifically the disk brake system, rather than the drum brake system.

Mechanical frictions of brakes causes high and diffuse particle emissions (1). High brake emissions are such as observed near traffic lights (2). The proportion of brake particles in urban air is doomed to growth unavoidably due to the exhaust emission controls become draconian (3).

Brake wear contributes between $16 \%$ and $55 \%$ by mass to total non-exhaust traffic related PM10 emissions in urban environments $(3,4)$. Regarding the PM 2.5 emissions in urban air, brake emissions offer between $39 \%$ and $63 \%$ of the non-exhaust traffic related particles $(5,6)$. The compositions of brake particles give rise to results dominated by $\mathrm{Fe} \mathrm{Cu}$, Ba under the main inorganic compounds commonly used in brake $(3,7)$ 
Due to the variations on results about the size and composition of brake particles, lab studies in the field of tribology are preferable to enable direct traceability between the sources and the emitted particles and to find the physical phenomena giving rise to emissions. Moreover, particle counting appears as the most relevant method to understand and characterize particles generated from brake wear (8).

Introduced in the 1950s, the disk brake system now remains the most common technique for braking on Internal Combustion Engines (ICE) cars. The principle is to have discs directly connected to each wheel and the rotation of the wheel implies the rotation of the disc. When braking, two pads come to clamp against the disc, which leads to slow down the disc rotation. The wheel rotation speed decreases, the vehicle brakes. The contact between disc and pads is a studied in tribological system $(9,10)$.

A tribological system is known to respond differently considering the changes in several parameters such as pressure, temperature, humidity, the properties of materials in contact, the existence or not of lubricant, etc $(11,12)$. Tribological systems are commonly characterized by their coefficient of friction, which is the ratio of the resulting tangential force (friction force) and the applied normal force. In our example of a disc brake, the normal force leads to a 'biting' of the disc by the pads.

In the case of a braking system, friction converts mechanical energy into thermal energy (heating of the brakes), kinetic energy (vibration), chemical energy (reactions), tribofilm, and surface generation (particles). The non-exhaust braking particles were called resulting particles.. Particles with a diameter bigger than $10 \mu \mathrm{m}$ are too big to be aerosolized (13). Airborne particles can be classified by their size. Beyond $2.5 \mu \mathrm{m}$ in diameter, particles are categorized as coarse (C). From $2.5 \mu \mathrm{m}$ to $100 \mathrm{~nm}$, particles are considered as fine $(\mathrm{F})$, and below they are ultrafine (UF) (14).

Braking particles are generated via different microscopic phenomena. First, they can result from a mechanical process, where a small part of the material is detached due to the high strain. Those particles represent the broad majority of emitted particles. Studies did not result in a consensual size distribution but most results showed a bimodal distribution: one ranging from around $100 \mathrm{~nm}$, and the other at around $300 \mathrm{~nm}$ (5). Researchers also studied the massweighted size distribution of braking emission and all concluded in a unimodal distribution. The mode was observed from $0.1 \mu \mathrm{m}$ to $10 \mu \mathrm{m}$, with a mean value mode of $2-3 \mu \mathrm{m}$ (5). Alternatively, another mode can appear when particles are produced by nucleation after the evaporation of pad material. These phenomena will occur when the disc temperature reaches and exceeds a transition temperature between $160^{\circ} \mathrm{C}$ and $200^{\circ} \mathrm{C}(15-17)$. Recent studies even showed a volatile emission induced by heat alone $(18,19)$. However, this process remains minor as the transition temperature, the temperature of decomposition of the filler (phenolic resin), is not reached at driving conditions (20). New particles created are ultrafine, and rarely exceed 20 $\mathrm{nm}$ in diameter (18).

A pad is a mixture of frictional additives, fillers, binders, and reinforcing fibers. There is no "typical composition" as components chosen by brake manufacturers depend on future use. The traditional and most used material for the disc is cast iron however, alternatives exist on the automobile market with aluminum and ceramics discs (21). Researchers have developed several experimental methods over the last years to do experimentally simulating. However, emissions observed tend to differ from a method to another and it appears impossible to compare results (22). Table 1 summarizes the most described setups available in the literature and shows that instrumentation or sampling method changes from a study to another. Four different experimental setups are described in eight different studies (23-29). It is important to determine a standardized experimental method providing physical data to be able to compare 
results. This method has to be representative of real-life driving conditions, and result in a reliable and repeatable measurement. Real-life tests are an option lacking repeatability at short test intervals and being even much more challenging concerning debris sampling. The braking emission problem gives birth to several workgroups. The Particle Measurement Program by the United Nations Economic Commission for Europe (UNECEPMP) which is an informal working group aims to define a protocol to test brake emission $(3,20,30)$. The EU project LOWBRASYS and the international REBRAKE project (31-34) intend to achieve a deep understanding of wear mechanisms, to address the braking emission issue by developing a new materials and systems. Through the UNECEPMP work, a new emission protocol has been proposed and its use as a reference is nearly a consensus (20). The generation must be representative of real-life braking conditions. Regarding the needed reliable methods to measure and sample, only a minor agreement exists. So far, the protocol leading to a repeatable measurement with a clear correlation between events and measurement is not determined. This article discusses how to design experiments to get a relevant braking particle assessment. This article also lists the observed differences in the experimental conditions of systems described in the literature, their potential influence on measurement, and their role in the impossibility to compare results from a system to another.

Table 1 : Summary of the literature review about airborne braking emission setups

\begin{tabular}{|c|c|c|c|c|c|c|c|c|}
\hline Reference & Test stand & Flow rate & $\begin{array}{l}\text { Presence } \\
\text { of bend }\end{array}$ & $\begin{array}{l}\text { Loss } \\
\text { estimation }\end{array}$ & $\begin{array}{l}\text { Chamber } \\
\text { volume }\end{array}$ & $\begin{array}{l}\text { Average } \\
\text { residence } \\
\text { time }\end{array}$ & $\begin{array}{l}\text { Isokinetic } \\
\text { sampling }\end{array}$ & Instrumentation \\
\hline $\begin{array}{l}\text { Perricone et } \\
\text { al. (23) }\end{array}$ & $\begin{array}{l}\text { Closed disc } \\
\text { brake } \\
\text { dynamometer }\end{array}$ & $\begin{array}{l}1175 \\
\mathrm{~m}^{3} / \mathrm{h}\end{array}$ & $\begin{array}{l}\text { Yes, } 180 \\
{ }^{\circ} \text { with } \\
\text { high } \\
\text { curvature } \\
\text { ratio }\end{array}$ & $\begin{array}{l}\text { Between } \\
90.8 \% \text { and } \\
98.7 \% \\
\text { transport } \\
\text { efficiency for } \\
10 \mu \mathrm{m}\end{array}$ & $0.817 \mathrm{~m}^{3}$ & $\begin{array}{l}\text { Around } 3 \\
\text { seconds }\end{array}$ & Yes & DEKATI ELPI+ \\
\hline $\begin{array}{l}\text { Hagino et al. } \\
\text { (24) }\end{array}$ & $\begin{array}{l}\text { Closed disc } \\
\text { brake } \\
\text { dynamometer }\end{array}$ & $120 \mathrm{~m}^{3} / \mathrm{h}$ & No & $\begin{array}{l}\text { Not } \\
\text { communicated }\end{array}$ & $\begin{array}{l}\text { Around } \\
0.11 \mathrm{~m}^{3}\end{array}$ & $\begin{array}{l}\text { Around } 3 \\
\text { seconds }\end{array}$ & Unknown & $\begin{array}{l}\text { TSI } \\
\text { DustTrakTM II }\end{array}$ \\
\hline $\begin{array}{l}\text { Wahlström } \\
\text { et al. (25) }\end{array}$ & $\begin{array}{l}\text { Closed pin- } \\
\text { on-disc }\end{array}$ & $7.7 \mathrm{~m}^{3} / \mathrm{h}$ & - & - & $0.135 \mathrm{~m}^{3}$ & $\begin{array}{l}\text { Around } 1 \\
\text { minute }\end{array}$ & No & $\begin{array}{l}\text { TSI Ptrak, TSI } \\
\text { Dustrak,TSI } \\
\text { SMPS ; In } \\
\text { other studies, } \\
\text { DEKATI ELPI } \\
+ \text {, OPS, } \\
\text { FMPS, } \\
\text { TEOM,GRIMM, } \\
\text {... (30-32) }\end{array}$ \\
\hline $\begin{array}{l}\text { Wahlström } \\
\text { et al. (28) }\end{array}$ & Roadside test & - & - & - & - & - & No & $\begin{array}{l}\text { GRIMM and } \\
\text { TSI DustTrak }\end{array}$ \\
\hline $\begin{array}{l}\text { Kukutschová } \\
\text { et al. (29) }\end{array}$ & $\begin{array}{l}\text { Closed disc } \\
\text { brake } \\
\text { dynamometer }\end{array}$ & $1.5 \mathrm{~m}^{3} / \mathrm{h}$ & $\begin{array}{l}\text { Yes, on } \\
\text { two } \\
\text { different } \\
\text { sampling } \\
\text { lines }\end{array}$ & $\begin{array}{l}\text { More than } 80 \\
\% \text { of transport } \\
\text { efficiency for } \\
10 \mu \mathrm{m} \text { in both } \\
\text { sampling } \\
\text { lines. }\end{array}$ & Unknown & - & No & $\begin{array}{l}\text { TSI SMPS, TSI } \\
\text { APS, BLPI }\end{array}$ \\
\hline
\end{tabular}

\section{Braking and tribology}

Braking emissions are the result of frictions, are thus in the scope of both tribological investigations and particle system analysis. It is necessary to agree on a common method to measure the distribution and the size of braking emissions to compare results between 
researchers. Setting a reference emission protocol is the first step for a common and repeatable protocol. The elaboration of a tribological test, source of the emission, has been subject to discussion for years $(20,35)$. This chapter deals with different methods of a tribological tests in the laboratory. Tribological tests can vary upon two main parameters: used braking cycle and experimental setup method to simulate braking.

\subsection{Braking cycle}

Braking cycles are a predefined series of precise deceleration rate, with nominal initial and final velocities. Braking cycle tests are initially designed as efficiency tests of the brake system, to make sure durability and safety during normal driving conditions. Those efficiency tests are composed of several strong braking events, eventually performed at high temperatures. The most known efficiency test is AK-Master (36). Some researchers used the AK-master procedure or part of it, for braking emission investigation (37). AK-Master requires several bars brake events (close to $8 \mathrm{~m} / \mathrm{s}^{2}$; real deceleration value depends on vehicle characteristics). For this reason, AK-Master procedure or other efficiency ways have been rapidly pointed out as not representative of real driving conditions. New cycles with lower deceleration rates (around $\left.2,5 \mathrm{~m} / \mathrm{s}^{-2}\right)$, appeared and substituted to efficiency tests $(20,38)$. Many cycles were developed. Perricone et al. (19) summarized some of them in an article, e.g. BSL-035, JC08, JASO C42788, SAE J 2707.

By means of an illustration, we display here a part of the procedure BSL-035. More procedures can be found described in detail elsewhere and would exceed the purpose of this contribution:

1. Reaching a disc rotation, representing around $50 \mathrm{~km} / \mathrm{h}$

2. Braking till the disc stops, with a specific deceleration of $2,94 \mathrm{~m} / \mathrm{s}^{2}$

3. Eventually waiting for a disc to cool down.

4. Accelerating to the same high disc rotation and repeat.

This procedure, closer to reality than safety tests, is meant to represent city driving. However, this cycle is still said to be not yet representative enough. Even though this iteration represents a part of a city driving but it does not apply to most cases, and overall, to other driving styles (i.e. country, highway, etc.).

In 2014, other authors proposed a further cycle (35). This test cycle, based on SAE J2707 method B, is composed of nine "blocks". Each of those blocks represents a specific driving style: highway, town, country road, descent, etc. Dividing cycles by blocks is a well-known method, used for examples in the assessment of wear characteristics of the disc brake system. In this case, those cycles are called Block Wear Evaluation (BWE). The cycle proposed by Alemani et al. (35) introduced a burnish block, which was supposed to remove the surface layers of the disc. During material stocking this surface treatment can limit oxidation and does not show the overall disc composition. The burnish block is a new step forwarding the representative of real emission. Still, this test cycle is judged as not representative enough for most of the researchers (35). Driving style can be considered as too regular, and again those blocks only represent a few percent of real braking.

The best way to get a representative testing series is to use an actual driving pattern. The Los Angeles City Traffic (LACT) cycle remains one of the most known cycles $(20,30)$. A car equipped with various sensors has been driven through Los Angeles several times. With the same idea of usual BWE cycles, the driven car crossed different roads, such as country road, city, highway, etc. Data collected led to the elaboration of the LACT cycle: a $24 \mathrm{~h}$ (3542 stops) cycle with different parameters recorded such as velocity, deceleration, or brake duration. Still, the LACT cycle turns out to be specific to a driving style but also too long. 3 hours long version of the LACT cycle has been proposed by the LOWBRASYS group and is widely used (30). 
The roads, cars, and driving regulations also its environments differ in different parts of the world(50). Considering the driving habits around the world, driving data has already been collected for the exhaust emission problem (39). This data was used to determine an exhaust test cycle called the Worldwide harmonized Light vehicles Test Procedure (WLTP) $(39,40)$. In 2018, a new cycle was proposed (20), which was based on the deisgn of WLTP data and is supposed to be representative of driving habits. This cycle last $4 \mathrm{~h} 24$ for 303 stops (maximum deceleration rate of $2,2 \mathrm{~m} / \mathrm{s}^{2}$ ). Different cooling rates are used depending on study conditions. As the temperature has a significant impact on the emission rate $(3,16,40)$, measurements observed are not often comparable from one system to another. Once a cycle has been chosen, the tribological system will perform, and it has to be defined as well. It is then also necessary to find the best option to simulate the contact between disc and pad in reliable conditions.

\subsection{Inertia dynamometer bench}

The inertia dynamometer bench is an instrument able to simulate contact of braking pads and brake discs. This device is made for experimentally simulating the friction between braking materials. For this reason, the most researchers are investigating the braking particles with a dyno $(23,24,29,41,42)$. In a dynamometer, real braking materials are used. A disc rotates with set inertia, representing the mass of the vehicle simulated. Two pads come to clamp against the disc to slow down the disc. This operation is similar to what happens in a real car. Several parameters are controlled such as initial speed, final speed, initial rotor temperature, braking deceleration, number of stops. It is possible to choose the disc rotation velocity, the inertia of the disc, the pads pressure, and the brake duration. All parameters of the WLTP cycle can be applied. The dynamometer bench stays as the best option but it is more expensive.

\subsection{Pin-on-disc}

Another option used for experimentally simulate the braking friction is pin-on-disc set-up. Pinon-disc sets a contact between a pin and disc. The pin is a small cylinder cut off of a pad, so they have an identical composition. The diameter of the pin has been chosen depending upon the contact pressure to apply. Pins must have a small friction surface to apply adequate normal stress with only a low force. Diameters of pins are usually less than a centimetre (17). The pin friction area is less than a percent of an actual pad surface.

Pin-on-disc is a piece of common equipment for experimentally simulate the friction contact between the materials. It is commonly used to determine the coefficient of friction, the quality of lubricant, or to characterize tribofilm formation (43). Similarly to dynamometer benches, a disc rotates at a set speed. But contrary to these, the pad's size was reduced, which leads to applying pressure on the small surface of the disc material.. Pin-on-disc is basic system that do not include inertia and braking deceleration concepts.

In dyno systems, pads clamp opposite sides of the disc, which balances forces and prevents the risk of distortion. The set-up of the pin-on-disc makes the clamping of the disc impossible (cf. Figure 1). It only slides on one side of the disc. 


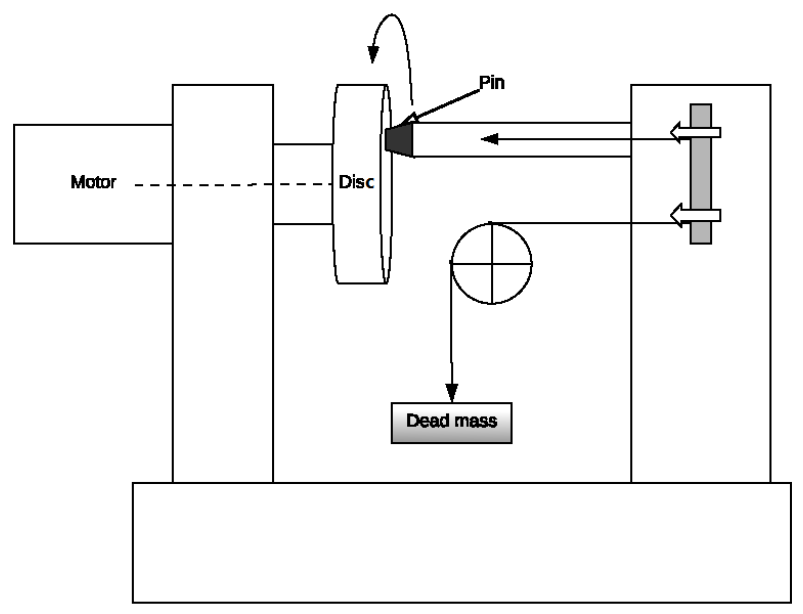

Figure 1: Scheme of a pin-on-disc

Studies have shown the relevancy of pin-on-disc in braking particle investigation $(25,44)$. Affordable and easy to use, pin-on-disc seems to be a good option for braking studies.

\subsection{On-road tests}

On-road tests are also a method to simulate braking emission $(28,44,46)$. On-road tests aim to assess the braking emission on a driving vehicle. Hence, the friction materials and airflow conditions are similar to driving conditions. This experiments investigates braking for the brake emission assessment because of the difficulty involves in the collection of emitted particles. So, these studies will remain rare due to its high level of contamination. External parameters such as temperature, humidity, or wind are difficult to control. Those parameters can cause significant tribological and airflow disturbances. WLTP cycle has been elaborated to represent worldwide driving conditions. When on-board tests are not done on the track, the equipped car has to obey traffic rules. But, the WLTP cycle is difficult to apply in real-life conditions like urban traffic.

\subsection{Cooling}

Temperature has a clear impact on on brake emission $(3,16,30,40,42)$. Friction in braking systems is a strong source of thermal energy. Therefore, discs are so designed to ensure their viability.

While on-road tests are directly cooled by air, like in real-life conditions, pin-on-discs and dynamometers need a cooling system. Brake discs are engineered to be good heat dissipaters. They are thermally conductive and designed with holes and ventilation channels. However, the system is closed and, air flow is still necessary to cool it. Flow rate leans on the cooling capacity of the system. For the same geometry, higher flow rates induce a higher cooling rate, and so lower temperatures are observed. In the WLTP cycle, braking events are paired to corresponding temperatures (cf. part 1.1). An equivalent cooling system has not been defined and, the researchers are using different cooling air flow for their experiments. Hagino et al. use a flow rate of $30 \mathrm{~m}^{3} / \mathrm{h}$ to ensure instrument accuracy (47). Whereas, Gramstat et al. performed tests with a flow rate of $3450 \mathrm{~m}^{3} / \mathrm{h}$. This discrepancy indeed could influence the emission rate, but it also changes the whole air flow, by increasing the turbulence intensity $(31,42,48)$. 


\section{Generated particles representativeness and confinement}

Over the last years, several authors $(23,47,49,50)$ studied the sampling part of the measurement. Yet no consensus on a proper reliable and, the repeatable protocol has been reached. The repeatability of the sampling protocol lays mainly in the representativeness of the particle flow. Emitted and measured particle distributions should be similar; if not, particle losses have to be estimated to reduce them or, a correction can be applied to compensate for the effects to trace the emitted distribution from the measured one. Finally, it is necessary to consider the impact of the time transport of the sampling method on the representativeness.

\subsection{Aerosol representativeness}

Aerosol characterization instruments cannot analyze a large quantity of air and, only a small fraction of generated particles are analyzed. In the field of braking measurement, the ELPI flow rate corresponds to $10 \mathrm{~L} / \mathrm{min}\left(0,6 \mathrm{~m}^{3} / \mathrm{h}\right)$ (cf. part 3). Compared to the actual cooling flow rate of the chamber (average of $1.10^{3} \mathrm{~m}^{3} / \mathrm{h}$, cf. part 1.5), this flow appears low. For representativeness and repeatability matters, this sampling flow must be representative of the whole emitted particles. To quantify the representativeness of the particles in the pipe, several air samples are collected through different places.

Some laboratories assess the representativeness of their system (51). Those investigations are only relatable to the specific air flow performances of the used dynamometer bench. All the dynamometer chambers used for braking particle investigations differ in terms of flow rate, geometry, or isokinetism. Representativeness can be achieved through different methods.

\subsubsection{Characterization of diffuse emission}

A sampling system aims at collecting a representative part of the aerosol as efficiently as possible and then transports it through tubing or a sample line to an instrument to characterize it (see fig 2). The global efficiency is expressed as the fraction of the aspirated aerosol through the opening inlet concerning particle available in the source and cumulates with transport efficiency through the sampling line. At that stage, two critical points are addressed

1. the inlet efficiency defined as the part of particles sampled and introduced in the inlet concerning particle available in the source and

2. the transport efficiency which is the part of the particles introduced in the inlet and reaching the instrument to the available particles at the opening inlet (52).

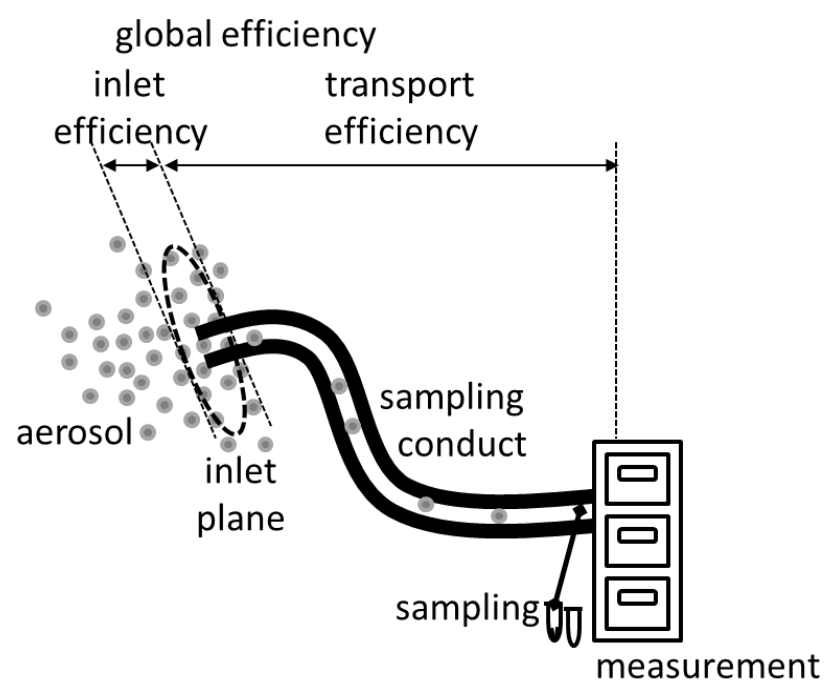

Figure 2 : Measurement of diffuse emission 
In the field of aerosol physics, particle behavior is mainly driven by their size. Very small particles will follow the streamlines and will be easily aspirated through the inlet plane with high efficiency. However, larger particles like supermicronic ones are driven notably by inertia forces and then weakly influenced by flow rate changes. Thus, most of the reasons that prevent representative sampling deal with the aerosol particle size. In general, larger particles are more actively influenced by mass forces such as gravitational and inertial forces, making a representative sampling more difficult whereas, smaller particles like submicronic ones or nanoparticles with higher diffusion coefficients are more easily lost to the walls of the sampling system. Generally speaking, the 8 main factors that can influence the aerosol characterization during the sampling are (52):

1. Aspiration efficiency and deposition in the opening inlet during sampling.

2. Deposition on the wall when the particles are transported through a sampling line.

3. Extremes (high or low) or variations in the ambient aerosol concentration.

4. Particle agglomeration during their passage through the sampling line.

5. Thermodynamically induced phenomena such as evaporation and /or condensation

6. Aerosolization of particles which were provisionally deposited on the walls

7. Local effects such as throttling by built-ups in the conduct

8. The effects of inhomogeneities of the particle concentrations in the conduct are due to the result of lacking isokinetism.

Existence of wear with metallic materials, two other factors can be added:

1. charged particles can be generated by mechanical frictions and interact with the near electric fields and on the inside of the inlet bring about a bias in sampling

2. chemical changes due to redox reactions caused by mechanical stress or compound reactivity accentuated by size decrease and, it can transform the generated aerosol during sampling time and transport.

All these factors are based on physical laws and, the main of them are detailed in the following sections of this chapter. Nevertheless, In the field of particles generated by braking, the difficulties are accentuated because of the severe conditions in temperatures, mechanical stress and, inconstant concentration observed during their production. In that case, great attention should be paid to the means of keeping the original aerosol during the sampling and transport process to have a relevant characterization.

\subsubsection{Isokinetism}

Representative collection and reliable transport of a diffuse aerosol from a source to characterization equipment is reported as a common difficulty and, achieving an accurate description of the emitted aerosol are often described by literature as a challenge (52). An aerosol sampling system consists of:

1. the particles are introduced in the opening intet of the measurement chain using a suction system for extracting the aerosol sample from its ambient environment,

2. a sample conduct tube to convey the aerosol sample to the measuring instruments, to a collection or a storage system, and, of course,

3. the instruments or samplers making the aerosol characterization possible.

Introduced as a concept of aerosol sampling in the 1950s, $(53,54)$, isokinetic sampling allows for sampling an aerosol without changing its properties, especially its size/mass distribution. 
The flow containing particles shall not be biased by sampling air flow, i.e. setting the sampling velocity to the level of the flow's velocity. (Figure 3).

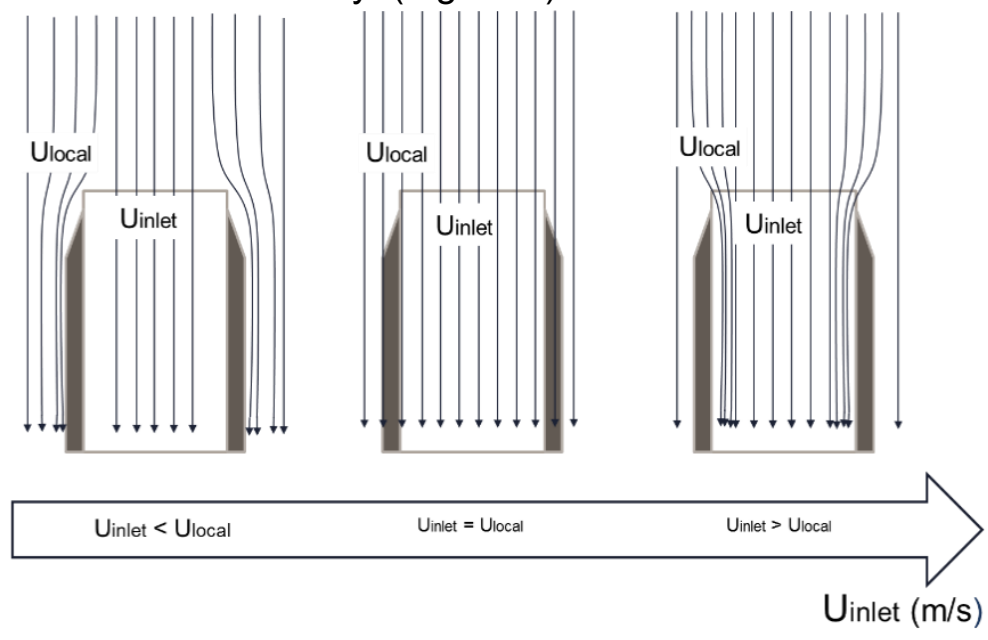

Figure 3 : Illustration of isokinetic sampling

Velocity differences can be a source of more or less fine or big particles in the sample flow, which means a shift of the size/mass distribution. The disturbances can be a source of bias in the distribution measurement. This bias can be understood via a dimensionless value: the Stokes number. The Stokes number indicates the ability of a particle to follow fluid streamlines. It can be calculated thanks to the following equation $(55,56)$ :

$$
S t k=\frac{1}{18} \frac{\rho_{p} d_{p}^{2} U}{l_{0} \mu_{g}}
$$

With Stk, the Stokes number, no unit,

$\rho_{p}$, the volumetric mass of the particle, in $\mathrm{kg} \cdot \mathrm{m}^{-3}$,

$d_{p}$, the aerodynamic diameter of the particle, in $\mathrm{m}$,

$U$, the fluid velocity, in $\mathrm{m}_{\mathrm{s}} \mathrm{s}^{-1}$,

$l_{0}$, the characteristic dimension of the pipe (usually the diameter of the pipe), in $\mathrm{m}$,

$\mu_{g}$, the fluid dynamic viscosity, in $\mathrm{kg} \cdot \mathrm{m}^{-1} \cdot \mathrm{s}^{-1}$.

The larger the Stokes number is, the more a particle is driven by its inertia, and the less the particle tends to follow the fluid's streamlines. For Stk $\ll 1$, particles follow streamlines; meanwhile for Stk >> 1, the particles are less driven by the flow. Sampling with a velocity lower than the flow velocity can lead to underrepresent small particles. Thus, a drift occurs during the measurement. Dennis et al. (53) stated that "The magnitude of error as reported in the literature depends upon the particle size and ranges from 10 to $20 \%$ for a $20 \%$ deviation from the isokinetic flow.".

To implement isokinetic sampling in a system, the inlet section must be calculated to respect the equation (2):

$$
\emptyset_{\text {inlet }}=\emptyset_{\text {local }} \sqrt{\frac{U_{\text {inlet }}}{U_{\text {local }}}}
$$


With $\emptyset_{\text {inlet }}$, the diameter of the inlet section in $\mathrm{m}$,

$\emptyset_{\text {local }}$, the diameter of the duct where the air is sampled, in $\mathrm{m}$,

$U_{\text {inlet }}$, the sampling velocity, often set by the instrument, in $\mathrm{m} . \mathrm{s}^{-1}$,

And $U_{\text {local }}$, the velocity of the fluid in the pipe, in $\mathrm{m}^{-\mathrm{s}^{-1}}$.

The inlet section must be designed to reach the appropriate sampling flow velocity. Researchers normally used a classical inlet section with a fixed diameter. It implies that the flow velocity if the sampling flow rate is constant and it cannot vary over time. In real-life conditions, the air flow around the brake system varies over time, and adjusting air flow over the experiment could be a new step forward into the representativeness of braking emission. If such variable air flow is observed, the inlet section flow would have to vary as well.

\subsection{Sampling efficiency}

Particle losses during transport are an inevitable part of particle flow experiments, but they have to be quantified and minimized. These losses depend on several parameters such as transport duration, flow velocity, particle diameter, environment properties, transport road, chemical properties of particles and tubes, etc (52). Braking events cause emissions of particles with a wide range of diameters, and this range constitutes the main challenge of particle losses control.

\section{External and internal contamination}

The adequate confinement of the set-up is evaluated by the ratio of signal to the background noise. This ratio is a function of the presence of further external particle sources, instrumentation uncertainties, emission levels, sampling, and transport efficiencies. In most experimental setups includes an over-pressurized chamber, a HEPA 14 filter is chosen for its high efficiency.

4. Losses

After their emission, particles need to be brought to the instrumentation. However, several particle loss phenomena can reduce transport efficiency. Every element of the system has a chamber, a sampling pipe connected to instrumentation induces a risk of loss. This chapter presents the losses of particles in air flow. Estimating sampling loss impact is an important task to characterize the originally emitted aerosol. Loss estimation has been obtained with oleic acid monodisperse aerosol. Park et al. (57) reported that polydisperse aerosol highlights a significantly higher wall deposition rate compared to a monodisperse solution.

\subsubsection{Diffusion loss}

Brownian motion of particles is a stochastic movement due to collisions of particles with fluid molecules $(52,58)$. The smaller the particles are, the more they are affected by collisions. The diffusion coefficient of gas influencing particles can be theoretically estimated as followed.

Here $D$, is the diffusion coefficient in $\mathrm{m}^{2} / \mathrm{s}$,

$$
D=\frac{k T C_{c}}{3 \pi \mu_{g} d_{p}}
$$

$\mathrm{k}$, the Boltzmann constant equal to $1,38 \cdot 10^{-23} \mathrm{~J} \cdot \mathrm{K}^{-1}$ (or $\left.\mathrm{kg} \cdot \mathrm{m}^{2} \cdot \mathrm{s}^{-2} \cdot \mathrm{K}^{-1}\right)$

$\mathrm{T}$ the temperature in $\mathrm{K}$,

$C_{c}$ a slip correction factor,

$\mu_{g}$, the dynamic viscosity of the fluid in $\mathrm{kg} \cdot \mathrm{m}^{-1} \cdot \mathrm{s}^{-1}$,

$d_{p}$, the particle diameter in $\mathrm{m}$. 
In brake emission investigations, air pressure and temperature can barely differ from standard conditions. Thus, in equation (3) the slip correction factor $C_{c}$ and can be estimated with the formula (4) (57):

$$
C_{c}=1+\frac{2 \lambda}{d_{p}}\left(1.246+0.418 \exp \left(-\frac{0.867 d_{p}}{2 \lambda}\right)\right.
$$

Here, $\lambda$ is the mean free path length of the gas molecules in $m$ Now the coefficient of diffusion D, and the Sherwood number Sh are defined. It is possible to estimate transport efficiency $\eta_{\text {tube,diff }}$ regarding diffusion loss with the following formula:

$$
\eta_{\text {tube,diff }}=\exp \left(-\frac{\pi D L S h}{Q}\right)
$$

Here $Q$, is the volumetric fluid flow in $\mathrm{m}^{3} . \mathrm{s}^{-1}$

$\mathrm{L}$, the lenght of the duct in $\mathrm{m}$

In most cases, those losses are theoretically highly negligible. The main reason is the high flowrate equipment developed by research groups. It substantially decreases the diffusion time of particles, which are less subject to encounter a wall in this period.

The Sherwood number is calculated as follow:

$$
S h=0,0118 \operatorname{Re}^{7 / 8} \cdot\left(\frac{\mu_{g}}{\rho D}\right)^{1 / 3}
$$

With Sh, the Sherwood number; and Re, the Reynolds number (cf. equation 9).

Sh is the mass transfer coefficient $(59,60)$. Like many other fluid mechanic parameters, it is possible to avoid complex computation thanks to an approximating formula (61).

\subsubsection{Losses due to impaction and inertia forces}

The first kind of loss is caused by bends in pipes. The transport to the instruments is usually done via a pipe, which in case of spatial difficulties must be bent. The impact of bends on transport efficiency is highly described in the literature $(49,55,56,62-70)$. Agudelo et al. even proposed different transporting design to minimize losses. Cheng and Wang's (64) computing method applies to laminar flow, whereas the method of Pui et al. (67) is relevant to turbulent flow. Regarding bent pipes, Pui et al. declared that "the most important parameters are Stokes number and the flow Reynolds number if particle motion is in the Stokesian regime $\left(\operatorname{Re}_{p} \leq 1\right)$ " and proposed by to estimate the transport efficiency through a bent pipe as a function of Stokes number:

$$
\eta_{\text {bend }}=10^{-0,963 . S t}
$$

With $\eta_{\text {bend }}$, the transport efficiency and St the Stokes number. Equation (7) is the most relevant computing method for most of the researcher's equipment. Computing with higher precision is possible but, is time and cost consuming and consequently rarely performed.

A similar mechanism appears when the pipe section of the pipe decreases. The contraction of the pipe implies a change in the direction of the external streamlines. It results in particle loss in external streamlines. Like bent tubing, contraction tubing affects mostly particles with Stokes number greater than 1. In 1996, Muyshom et al. proposed the empirically found relation (8) to estimate transport efficiency through a tubing contraction $\eta_{\text {cont }}(71)$ : 


$$
\eta_{\text {cont }}=1-\frac{1}{1+\left(\frac{2 * \operatorname{Stk}\left(1-\left(\frac{A_{0}}{A_{i}}\right)^{2}\right)}{3.14 . \exp \left(-0.0185 . \theta_{\text {cont }}\right)}\right)^{-1,24}}
$$

Where $A_{0}$ and $A_{i}$ are respectively the cross-sectional area before and after the contraction, and $\theta_{\text {cont }}$ is the half angle of the contraction. The equation is only valid when $0.001 \leq \operatorname{Stk}\left(1-A_{0} / A_{i}\right) \leq 100$ and $12^{\circ} \leq \theta_{\text {cont }} \leq 90^{\circ}(71)$.

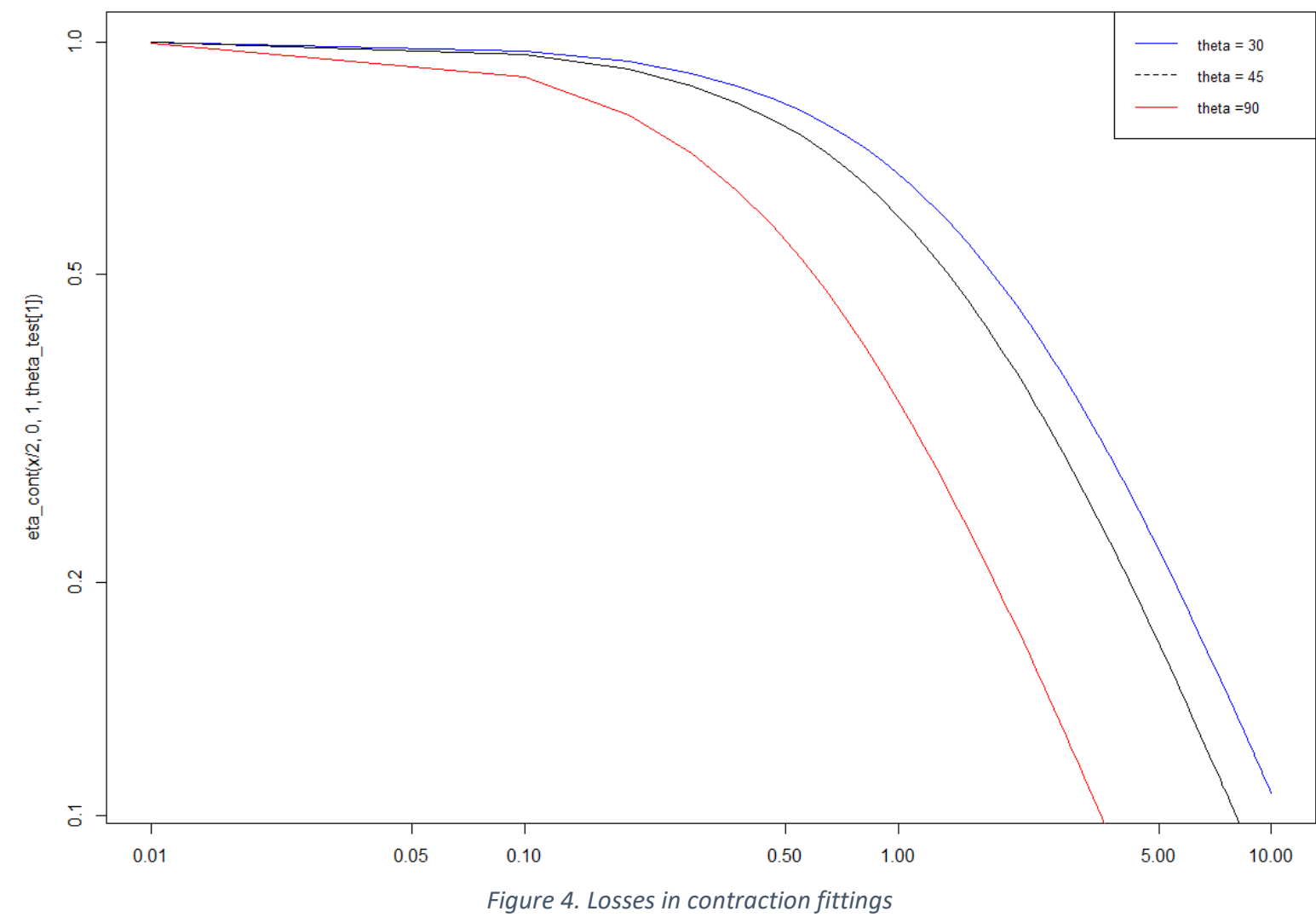

Enlargement of the pipe section does not induce inertia losses as there is no obstacle to particle trajectory. However, vortices appear when the angle of enlargement is superior to $8^{\circ}$ (or a half angle of $4^{\circ}$ ) (72). By curving streamlines toward the pipe walls, those vortices are a potential source of loss, indirectly caused by the enlargement. There is currently no model to estimate those enlargement losses. For this reason, the needed enlargement must be designed with an angle inferior to $8^{\circ}$ to ensure loss control.

Once a particle is generated, it is moved in a tangent direction of the disc. This effect is particularly high on walls near the friction area. A study of the CFD (model) has been performed on this topic. Determining the overall behavior of particles in the chamber appears to be complex for now. The air flow for each chamber can be changed with disc speed and it is difficult to estimate the influence of disc speed in the chamber.

\subsubsection{Turbulence losses}

Another type of losses is those caused by turbulences (73-76). Reynolds number described in Equation 8 gives the ratio of inertial forces to viscous forces in a fluid. The flow profile is predicted by this ratio $\mathrm{Re}$ and can be laminar or turbulent. (52). In a turbulent flow, turbulences or vortices of various sizes punctually appear and disappear resulting in turbulent diffusion. (77). 
With Re, the Reynolds number

$$
R e=\frac{\rho U L}{\mu_{g}}
$$

$\rho$, the volumetric mass in $\mathrm{kg} \cdot \mathrm{m}^{-3}$

$\mathrm{U}$, the characteristic fluid speed in $\mathrm{m} . \mathrm{s}^{-1}$

$\mathrm{L}$, the characteristic linear dimension of the pipe (or else) in $\mathrm{m}$

$\mu_{g}$, the dynamic viscosity of the fluid in $\mathrm{kg} \cdot \mathrm{m}^{-1} \cdot \mathrm{s}^{-1}$.

Under a critical value of the friction Reynolds number named $\mathrm{Re}_{\mathrm{T}}$, generally estimated between 2000-3000, no turbulence appears and the fluid is laminarly flowing (78). Meanwhile, if $\operatorname{Re}>$ $\mathrm{Re}_{\mathrm{T}}$, the flow is turbulent. Equation 9 does not take into account local sources of variation of the parameters such as irregularities on the pipe wall or particles in the air flow. As a result, the limit between laminar and turbulent flow can change from one pipe to another. It is expressed by the friction Reynolds number $\left(\mathrm{Re}_{\mathrm{T}}\right)$. Turbulences are often generated close to walls, where fluid shear stresses are higher (78).

When the inertia of particles is high enough, particles penetrate the sub-layer of air near the wall and are hurting the wall (52). The transport efficiency $\eta_{t u r b}$ of particles passing through a pipe is calculated by equation (10):

$$
\eta_{t u r b}=\exp \left[-\frac{\pi \emptyset L U_{t}}{Q}\right]
$$

With $\varnothing$, the pipe section in $\mathrm{m}$;

$\mathrm{L}$, the length of the pipe in $\mathrm{m}$;

$U_{t}$, the turbulent inertial deposition velocity in $\mathrm{m}^{-\mathrm{s}^{-1}}$;

$Q$, the volumetric fluid flow in $\mathrm{m}^{3} \cdot \mathrm{s}^{-1}$.

The turbulent inertial deposition velocity represents the velocity necessary to a particle to cross the sub-layer and impact on the wall. Another expression of $U_{t}$ is given by the equation (11):

$$
U_{t}=U_{+} \times \sqrt{\frac{\tau_{0}}{\rho}}
$$

Where:

$U_{+}$is the dimensionless deposition velocity;

$\tau_{0}$ is the fluid shear stress in $\mathrm{kg} \cdot \mathrm{m}^{-1} \cdot \mathrm{s}^{-2}$;

$\rho$ the volumic mass of the fluid in $\mathrm{kg} \cdot \mathrm{m}^{-3}$.

The formulas for the dimensionless deposition velocity is not properly defined. $U_{+}$has only been approached by several approximated curve equations as a function of $\tau_{+}(52,73,76) . \tau_{+}$ is the dimensionless particle relaxation time, highly related to Stokes number :

$$
\tau_{+}=S t k \frac{\tau_{0} \cdot l_{0}}{\rho \cdot U^{2}}
$$

All parameters are previously introduced in equation (1) and (11). $U_{+}$is described as a function of $\tau_{+}$. Literature concludes that the transport of particles is more efficient when $\tau_{+}$is close to 0.14 . 


\subsubsection{Settling losses}

Settling losses are a direct result of gravity on particles and are predominant for particles with a diameter greater than $1 \mu \mathrm{m}$ (see equation 5). Particles tend to settle over time. Sedimentation velocity is proportional to the square of the particle diameter (Stokes Law) (52):

$$
\beta_{\text {set }}=\frac{U_{\text {set }}}{h} \cdot \tau_{\text {set }}
$$

With $\beta_{\text {set }}$, the losses due to settling (no unit),

$\tau_{\text {set }}$, the duration of dropping of a particle in chamber or pipe, in $\mathrm{s}$,

$U_{\text {set }}$, the terminal settling velocity in $\mathrm{m}^{-\mathrm{s}^{-1}}$,

$\mathrm{h}$, the chamber or pipe height in $\mathrm{m}$.

In the case of braking emission investigations, a cooling system is needed. After the emission, the aerosol generated in the chamber is evacuated in a pipe. The settling behavior of the system is described by the gravitational settling parameter $Z$ (no unit), which is computed as follows (52):

$$
Z=\frac{\pi L}{4 Q} U_{\text {set }}
$$

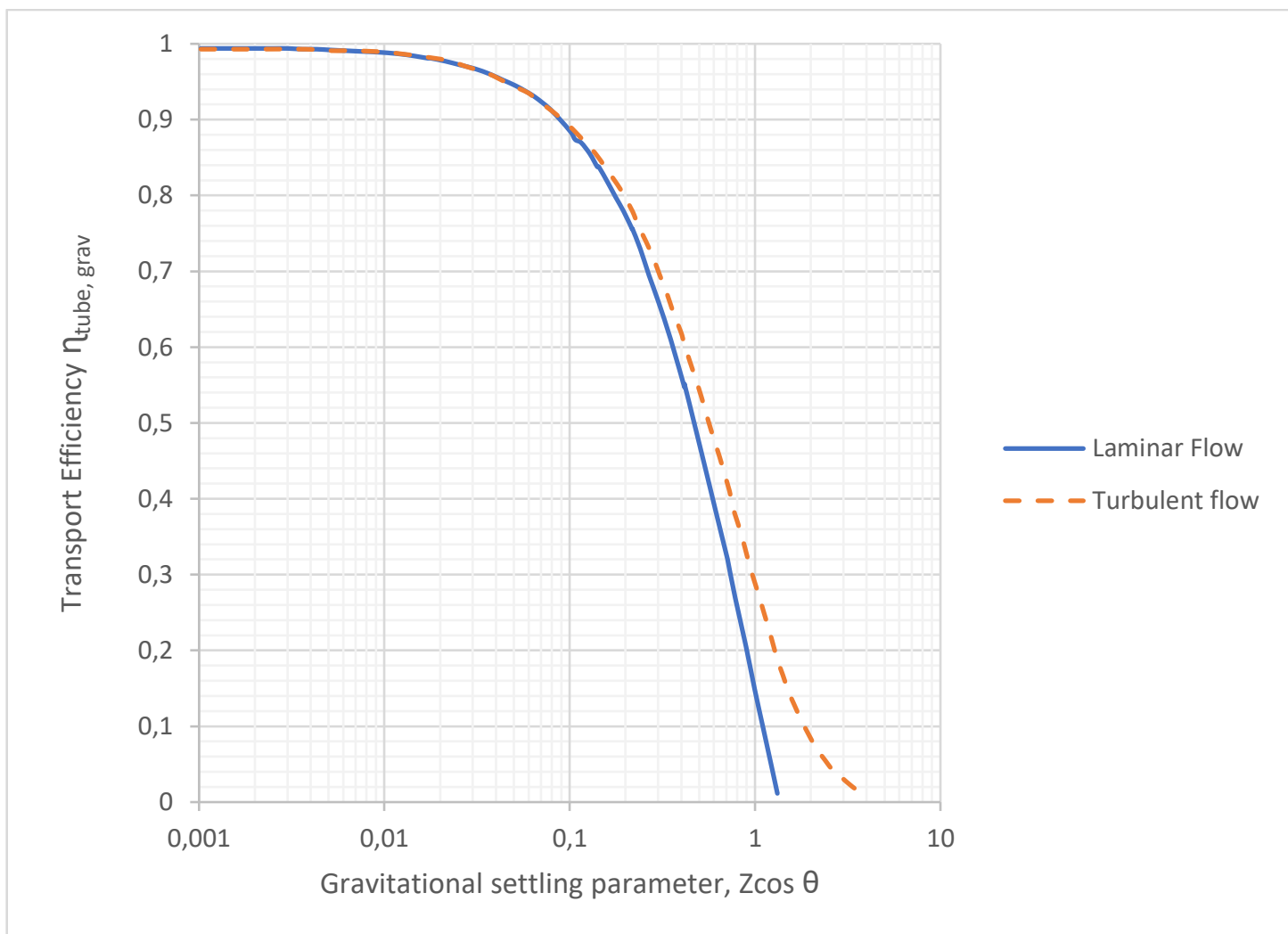

Figure 5: Gravitational settling in a pipe with inclination $\vartheta$

Settling losses can occur during transport in the pipe depending on the flowing nature (cf. Fig. 5). Figure 5 shows the evolution of transport efficiency of particles flowing through a tube inclined at an angle $\theta$ as a function of the gravitational settling parameter $Z$ (52).

\subsubsection{Computational approach for loss assessment}

Many aerosol measurements need to be transported through tubing to make possible a characterization. These set up need a loss assessment to estimate the assessment limits of 
sizing, counting, and sampling. Von der Weiden, et all develop a software tool named Particle Loss Calculator, (PLC) able to determine aerosol sampling efficiency and particle transport losses due to passage through tubing (72)

The software takes into account different aspects of loss to give a relevant particle loss assessment and includes the most important sampling and transport effects like a variation on tubing, flow rate, bending. Other points are treated by software like non-isoaxial and nonisokinetic aerosol sampling, aerosol diffusion and sedimentation which interest the braking particle sampling (72). Thus, aerosol sizing obtained by equipment could be corrected by this loss estimation and could lead to a more precise description of the original brake particles emitted near the friction contact.

\subsubsection{Chamber design}

\subsubsection{Response time}

Response time is defined here as the elapsed time between the emission of particles and their measurement. In a case of high response time, the last particles emitted from the first event can be measured with the first particles emitted from the second event. This crosscontamination can have different effects depending on the instrumentation and their own sampling frequency. The paragraph here reminds the necessity to prefer low response time. The notion of response time is a direct result of the residence time of particles in the chamber (23). The difficulty to anticipate the air motion in the chamber causes trouble to estimate losses (79). Some work was performed in controlled conditions (80-82) but, there is no theoretical model that has been found yet. The residence time of particles in the chamber can be estimated by the time of renewal of the chamber air (50) :

$$
\tau_{\text {chamber }}=\frac{Q_{\text {total }}}{V_{\text {chamber }}}
$$

With $\tau_{\text {chamber }}$, the complete renewal chamber air in $\mathrm{min}^{-1}$

$Q_{\text {total }}$, the total inlet or outlet flow in $\mathrm{m}^{3} \cdot \mathrm{min}^{-1}$

$V_{\text {chamber }}$, the volume of the chamber in $\mathrm{m}^{3}$

This estimation showcases two approaches to increase complete renewal chamber air:

On the one hand, it is possible to lower the chamber volume. In general, the literature shows that the chamber size is under $0,817 \mathrm{~m}^{3}$ (38) with an average value under $0,2 \mathrm{~m}^{3}$. However, the main issue of a small chamber is the inevitable impaction inertia losses due to the motion of particles directly on the wall after their emission from the brake system (cf. part 2.2.2.2.).

On the other hand, increasing the total flow of the experiment is also a solution. Researchers design chambers with different flow values from $7.7 \mathrm{~m}^{3} / \mathrm{h}$ to $3450 \mathrm{~m}^{3} / \mathrm{h}$, with an average value of around $10^{3} \mathrm{~m}^{3} / \mathrm{h}(16)$.

Response time can be changed in various ways that were all detailed in the previous part: decreasing chamber size, increasing flow rate, and decreasing pipe section can lower the time between emission and measurement. However, the time between emission and measurement has not been defined yet; it is an open question. In real conditions, the average time where a particle stays airborne is unknown and is impossible to define. Physical phenomena, such as nucleation or aggregation $(16,83)$ occur but, it is impossible to know their impact. 


\subsubsection{Electrostatic deposition and thermophoretic effect}

Researchers chose two different materials: steel or acrylic glass to confine set-up. Steel is the most used material $(38,49$, and few authors use acrylic glass (25). Steel is a conductive material, which avoids electrical charges and limits the electrostatic deposition of charged aerosol. Indeed, studies have shown the impact of using different materials on wall deposition $(56,68,84-87)$.

The thermophoretic deposition is another common loss of particles $(88,89)$. If a wall is colder than ambient air, thermophoretic deposition can be observed. The gradient of temperature in a gas can lead to the migration of particles to the colder area. Those effects are difficult to assess.

\subsubsection{Geometry and layout of the chamber}

The best place for the tribological contact, the orientation of disc, location of inlet, and outlet of the chamber shall be defined to minimize particle losses.

The proximity of walls near disc and pads is a high source of loss. Hence except for one case (51) where the chamber is large enough to assume that direct impaction is negligible, most of the tribological contacts are placed in the center of the chamber $(7,23)$.

In the case of the axial orientation of the disc, it is usually vertical, like real-life braking conditions. At least one group works with a horizontal disc (90).

\section{Number characterization of generated aerosols}

\subsection{Aerosol Particle Sizer (APS)}

In Aerosol Particle Sizers, the particle aerosol flows through a cell with a laser beam. Particle in the cell diffuse light depending on their size and morphology (91). A photodetector converts the light pulses into an electrical signal. The number concentration and the aerodynamic diameter of the equivalent sphere can be determined with height and number of pulses based on Mie's theory (92). The particle's refraction index must be known. Those instruments are used to determine size distribution between $0,5 \mu \mathrm{m}$ and $20 \mu \mathrm{m}$. This size range can be deemed as irrelevant for isolated nano-particle investigations. An APS measurement added to an SMPS measurement (see part 3.4.) can be used to assess aggregate and agglomerate impacts.

\subsection{Condensation nucleus counters (CNC)}

Condensation Nucleus Counters (CNC) or Condensation Particle Counters (CPC) are standard devices used to determine particle number concentration in an aerosol. Particles below $100 \mathrm{~nm}$ are unmeasurable by direct detection of laser counting. Particles are placed in a saturated environment, with a high vapor pressure liquid, usually butanol, isopropanol, or water. Vapors and particles are then cooled which, leads to condensation of vapors on the particle surface. This condensation enlarges particles. The enlarged particles are now detectable by the optical system placed after the condenser. This optical system is composed of a laser and two photodetectors. One photodetector is used as a background reference when no particle crosses the laser beam. The other photodetector catches the light diffused by particles. Several models of CNC exist and, their size range varies from one to another. For example, TSI CPC 3776 counts particles from $4 \mathrm{~nm}$ to $3 \mu \mathrm{m}$ while TSI CNC 3007 only counts from $10 \mathrm{~nm}$ to $1 \mu \mathrm{m}$. 


\subsection{Electrical Low Pressure Impactor (ELPI)}

ELPI selects solid particles by their inertia and then electrically detects them (93). Particles are charged with a corona charger before being introduced to the cascade impactor. Particles are then collected in a series of 13 impactors depending upon their size. Each floor is equipped with an electrical counting system. ELPI classifies particles with diameters between $7 \mathrm{~nm}$ and $10 \mu \mathrm{m}$ in 13 specific ranges. ELPI provides a simultaneous estimate at all channels, thus aerosol sizing can be assessed with a short response time of between 1 and 20 seconds. ELPI is not relevant for low concentration aerosols. The risk of bouncing of the particles can bias the sizing. Using the most sensitive range, ELPI has a detection limit of $130 \# / \mathrm{cm}^{3}$ at $0.05 \mu \mathrm{m}$ of diameter, $5 \# / \mathrm{cm}^{3}$ at $0.5 \mu \mathrm{m}$ and $0.4 \# / \mathrm{cm}^{3}$ at $5 \mu \mathrm{m}(94)$.

\subsection{Scanning Mobility Particle sizer Spectrometer (SMPS)}

SMPS is used to assess size distribution and number concentration of particles between 2,5 and $1000 \mathrm{~nm}$ (95). A classifier, DMA (Differential Mobility Analyser), selects particles by their size in a large number of channels. Aerosol firstly goes through an impactor that will retain the biggest particles. It passes through a radioactive source $(85 \mathrm{Kr}, 75 \mathrm{MBq}$ intensity) or through an X-ray source, called neutralizer, which charges particles with a high concentration of ions. The aerosol passes then in a column where an adjustable electric field is applied between two electrodes inducing a further motion of the previously charged object. This particle motion is proportional to their size, making possible the size selection of the particles.

Only the particles with a known diameter and charge are extracted from the DMA as a monodisperse aerosol.

The SMPS is the addition of a DMA and a CNC. The DMA is used to assess the size of particles but, the CNC counts the extracted particles to obtain concentration. The SMPS needs to browse size ranges from one after another with affecting time resolution. SMPS can determine aerosol sizing in 20 seconds minimum.

\subsection{Instrumentation comparison}

As described in previous parts, instrumentation use different operating procedures that can lead to different results (96-98). Even if instruments provide generally similar results in a controlled sampling setting (96). Divergences can be observed at low number concentration and the lower and upper working size ranges of the instruments (96). The type of particle measured can also lead to the measurement of some error depending on instrumentation (96). Price et al. showed, for instance, that FMPS identified bimodal distribution for $\mathrm{TiO}_{2}$ and fumed silica, whereas the distribution generated was unimodal (96). Table 2 resumes instrumentation used in braking particle domain, their working size, the type of particle diameter provided, the related studies, and the modes identified. It has to be kept in mind that all studies can differ in the experimental protocol but also in braking material used. Hence, number characterization cannot be discussed here as other parameters are also varying. The Resulting diameter type of Table 2 column is here to specify the outcoming diameters from instrumentation, which are not directly comparable. The aerodynamic diameter of a particle is meant to describe inertial properties in the gas. The aerodynamic diameter of a given particle is equal to the diameter of a spherical particle with a density of 1 (99). Electrical mobility is used to classify particles upon their ability to react to an electrical field. Algorithms are applied to determine which diameter is equivalent to given electrical mobility (95). 


\begin{tabular}{|c|c|c|c|c|}
\hline Instrument & $\begin{array}{l}\text { Operating particle } \\
\text { size range }\end{array}$ & $\begin{array}{l}\text { Resulting diameter } \\
\text { type }\end{array}$ & Studies & Number distribution \\
\hline ELPI/ELPI+ & $7 \mathrm{~nm}-10 \mu \mathrm{m}$ & $\begin{array}{l}\text { Aerodynamic } \\
\text { diameter }\end{array}$ & Sanders et al. & Unimodal $(1.0 \mu \mathrm{m})$ \\
\hline \multirow{3}{*}{ SMPS } & \multirow{3}{*}{$14 \mathrm{~nm}-730 \mathrm{~nm}$} & \multirow{3}{*}{$\begin{array}{l}\text { Electrical mobility } \\
\text { diameter }\end{array}$} & Kukutschová et al. & $\begin{array}{l}\text { Bimodal }(100 \text { and } 300 \mathrm{~nm}) \text { (rotor } \\
\text { temperature of } 300^{\circ} \mathrm{C} \text { ) }\end{array}$ \\
\hline & & & Wahlström et al. & Bimodal (280 and $350 \mathrm{~nm})$ \\
\hline & & & Wahlström et al. & Multimodal $(100,280,350$ and $550 \mathrm{~nm})$ \\
\hline \multirow{3}{*}{ APS } & \multirow{3}{*}{$0.523 \mu \mathrm{m}-20 \mu \mathrm{m}$} & \multirow{3}{*}{$\begin{array}{l}\text { Aerodynamic } \\
\text { diameter }\end{array}$} & lijima et al. & Unimodal $(1.0-2.0 \mu \mathrm{m})$ \\
\hline & & & lijima et al. & Unimodal $(0.8-1.0 \mu \mathrm{m})$ \\
\hline & & & Kwak et al. & Unimodal $(1.0-3.0 \mu \mathrm{m})$ \\
\hline \multirow{3}{*}{ GRIMM } & \multirow{3}{*}{$0.25 \mu \mathrm{m}-32 \mu \mathrm{m}$} & \multirow{3}{*}{$\begin{array}{l}\text { Aerodynamic } \\
\text { diameter }\end{array}$} & Wahlström et al. & Unimodal $(0.35 \mu \mathrm{m})$ \\
\hline & & & Wahlström et al. & Bimodal (280 and $350 \mathrm{~nm}$ ) \\
\hline & & & Wahlström et al. & Multimodal $(100-550 \mathrm{~nm})$ \\
\hline OPS & $0.3 \mu \mathrm{m}-10 \mu \mathrm{m}$ & $\begin{array}{c}\text { Aerodynamic } \\
\text { diameter }\end{array}$ & von Uexküll et al. & Unimodal $(0.5-1.0 \mu \mathrm{m})$ \\
\hline LSA & $0.04 \mu \mathrm{m}-262 \mu \mathrm{m}$ & $\begin{array}{c}\text { Aerodynamic } \\
\text { diameter }\end{array}$ & Mosleh et al. & Bimodal ( $350 \mathrm{~nm}$ and $2.0 \mu \mathrm{m})$ \\
\hline
\end{tabular}

Aerodynamic and electrical mobility diameter data can be merged using the effective density of the particle $(26,100)$. However, braking particle studies merging those diameters rely on a $5000 \mathrm{~kg} / \mathrm{m}^{3}$ volumic mass determined in 2003 by Sanders et al. using the density of the worn initial material $(25,101)$. This method approximates equivalent diameter and electrical mobility diameter to be equal. The equivalent diameter of a given particle is equal to the diameter of a spherical particle with the same density. In a forward study, the effective density of particles between 1,3 and $10 \mathrm{~nm}$ has been determined (26) and it can be used for nanostructured particles analysis when the morphology of braking particles will be more defined.

\section{Discussion and conclusion}

In the field of braking particle characterization, many parameters need to be taken into account. This article reviews the different challenges to be solved when designing a brake emission measurement system. Two systems can be used. Pin-on-disc bench is affordable and can simulate braking mechanisms. Since the cooling system is not needed because of the low thermal energy created. The particle transport airflow can be set independently of it and then it can be chosen to limit the transport losses or maximize concentrations measured. Its low emission level and the impossibility to implement vehicle inertia makes the pin-on-disc bench only able to relate qualitative observations. The large contribution of the understanding of particle generation and characterisation was performed on a pin-on-disc setup (25-27). 
Inertia dynamometer bench studies involve considerable investment. However, this system might become fundamental to future standardization as it could provide measurements in reallife conditions. The high thermal energy dissipated by the friction forces to use an airflow to keep the friction material cool. Inertia dynamometer bench can simulate real driving conditions. The representativeness of friction material temperatures can be achieved with respect to WLTP cycle (20). It has to be noted that WLTP disc temperature was determined on road, with a specific vehicle (inertia) and a specific braking system. The determined temperature cycle represents driving cooling conditions. The same specific parameters (inertia and braking system) can be used to fit the disc temperatures of the cooling system in the dynamometer bench. The bench can be used to investigate the other braking systems/materials using those same cooling conditions, which represent real cooling conditions.

Today, the representativeness of braking particle characterization is under question. Estimation losses can be computed with techniques exposed above. An important point to know about the losses concerns in the sampling pipe, where a high flow rate can be preferred to improve aerosol transport and reduce diffusion and settling losses. It should be pointed out that a too high velocity can lead to turbulence losses and a too-high flow rate leads to low particle concentrations. If the setup requires bending, a lower flow velocity can be set, either by increasing the inlet section or by decreasing the flowrate.

In a broader context, understanding and measurement of braking emission still lack several knoweldge gaps:

- Understanding of generation mechanisms has still to be developed. The origin of particles and their behavior in the contact is still an open question.

- Simulation of emission mechanisms is out of range for the moment because of the large surfaces to take into account and the complexity of pad compositions. Braking emission cannot be estimated by computing.

- Losses in the chamber are today difficult to estimate, and the best way to avoid these is to shorten the residence time of particles in the chamber.

- Instrumentation is used to assess the emissions changes from a study to another and can influence the data. A stabilized measurement protocol based on a consensus on equipment could increase the comparability of the results. A better characterization of particles would help to describe the link between the different kinds of diameters used by instrumentation.

\section{Acknowledgment}

The authors gratefully acknowledge our respective institutions for making this transdisciplinary project possible. Also, we acknowledge the financial support of the ANRT grant number in this project. The French Ministry for Ecology funding is gratefully acknowledged.

\section{Declaration of conflicting interests}

The author(s) declared no potential conflicts of interest with respect to the research, authorship, and/or publication of this article. 


\section{Funding}

The author(s) disclosed receipt of the following financial support for the research, authorship, and/or publication of this article: This work is supported by the ANRT for the grant number and the French Ministry for Ecology for the funding.

\section{Bibliography}

1. Yan F, Winijkul E, Jung S, et al. Global emission projections of particulate matter (PM): I. Exhaust emissions from on-road vehicles. Atmos Environ 2011; 45(28): 4830-4844.

2. Hulskotte JHJ, Roskam GD and van der Gon HD. Elemental composition of current automotive braking materials and derived air emission factors. Atmos Environ 2014; 99: 436445.

3. Grigoratos T and Martini G. Non-exhaust traffic related emissions. Brake and tyre wear PM. [Internet], https://core.ac.uk/download/pdf/38628016.pdf (2014, accessed25 May 2020).

4. Guerreiro C, Hora' lek J, de Leeuw F, et al. Air quality in Europe: 2015 report [Internet]. Luxembourg: publications Office. http://bookshop.europa.eu/uri?target=EUB:NOTICE: THAL15005:EN:HTML (2015, 4 November 2019).

5. Grigoratos T and Martini G. Brake wear particle emissions: a review. Environ Sci Pollut Res 2015; 22(4):2491-2504.

6. Thorpe A and Harrison RM. Sources and properties of non-exhaust particulate matter from road traffic: a review. Sci Total Environ 2008; 400(1): 270-282.

7. Hagino $\mathrm{H}$, Oyama M and Sasaki S. Laboratory testing of airborne brake wear particle emissions using a dynamometer system under urban city driving cycles. Atmos Environ 2016; 131: 269-278.

8. EEA E. Air quality in Europe - 2015 report. Report no. 5/ 2015, 2015. Copenhagen:

European Environment Agency.

9. Eriksson M, Bergman $F$ and Jacobson S. On the nature of tribological contact in automotive brakes. Wear 2002; 252(1): 26-36.

10. Eriksson $M$ and Jacobson S. Tribological surfaces of organic brake pads. Tribol Int 2000; 33(12): 817-827.

11. Halling J. Principles of tribology.Macmillan International Higher Education, 1978, p.414.

12. Bhushan B. Introduction to tribology. New York, NY: John Wiley \& Sons, 2013, p.672.

13. Williams MMR and Loyalka SK. Aerosol science: theory and practice, http://inis.iaea.org/Search/search.aspx?orig_q=RN:23024824 (1991, accessed 9 October 2019).

14. Liu L, Urch B, Poon R, et al. Effects of ambient coarse, fine, and ultrafine particles and their biological constituents on systemic biomarkers: a controlled human exposure study. Environ Health Perspect 2015; 123(6): 534-540.

15. Alemani M, Nosko O, Metinoz I, et al. A study on emission of airborne wear particles from car brake friction Pairs. SAE Int J Mater Manf 2015; 9(1): 147-157.

16. Nosko $\mathrm{O}$, Vanhanen $\mathrm{J}$ and Olofsson $\mathrm{U}$. Emission of $1.3-10 \mathrm{~nm}$ airborne particles from brake materials. Aerosol Sci Technol 2017; 51(1): 91-96.

17. Wahlstro $\cdot m$ J, Olander $L$ and Olofsson $U$. A pin-on-disc study focusing on how different load levels affect the concentration and size distribution of airborne wear particles from the disc brake materials. Tribol Lett 2012; 46(2): 195-204.

18. Ma J, Olofsson U, Lyu Y, et al. A comparison of airborne particles generated from disk brake contacts: 
induction versus frictional heating. Tribol Lett 2020; 68(1): 38.

19. Perricone G, Mata' jka V, Alemani M, et al. A test stand study on the volatile emissions of a passenger car brake assembly. Atmosphere 2019; 10(5): 263.

20. Mathissen M, Grochowicz J, Schmidt C, et al. A novel real-world braking cycle for studying brake wear particle emissions. Wear 2018; 414-415: 219-226.

21. Maleque MA, Dyuti S and Rahman MM. Material selection method in design of automotive brake disc. In: Proceedings of the world congress on engineering, London, UK, 30 June-2 July 2010, vol. III, p.5.Philippe et al. 13

22. Chan D and Stachowiak GW. Review of automotive brake friction materials. Proc IMechE, Part D: J Automobile Engineering 2004; 218(9): 953-966.

23. Perricone $\mathrm{G}$, Wahlstro $\cdot \mathrm{m} \mathrm{J}$ and Olofsson U. Towards a test stand for standardized measurements of the brake emissions. Proc IMechE, Part D: J Automobile Engineering 2016; 230(11): 1521-1528.

24. Hagino $\mathrm{H}$, Oyama $\mathrm{M}$ and Sasaki S. Airborne brake wear particle emission due to braking and accelerating. Wear 2015; 334-335: 44-48.

25. Wahlstro $\cdot \mathrm{m}$ J, So $\cdots$ derberg A, Olander L, et al. A pin-on disc simulation of airborne wear particles from disc brakes. Wear 2010; 268(5-6): 763-769. 26. Nosko O and Olofsson U. Effective density of airborne wear particles from car brake materials. J Aerosol Sci 2017; 107: 94-106.

27. Alemani M, Wahlstro - $\mathrm{m} \mathrm{J}$ and Olofsson $\mathrm{U}$. On the influence of car brake system parameters on particulate matter emissions. Wear 2018; 396-397: 67-74.

28. Wahlström $\mathrm{J}$ and Olofsson $\mathrm{U}$. A field study of airborne particle emissions from automotive disc brakes. Proc IMechE, Part D: J Automobile Engineering 2015; 229(6): 747-757.

29. Kukutschova' J, Moravec P, Toma' šek V, et al. On airborne nano/micro-sized wear particles released from lowmetallic automotive brakes. Environ Pollut 2011; 159(4): 9981006.

30. Mathissen M, Grigoratos T, Lahde T, et al. Brake wear particle emissions of a passenger car measured on a chassis dynamometer. Atmosphere 2019; 10(9): 556.

31. Nosko O, Alemani M and Olofsson U. Temperature effect on emission of airborne wear particles from car brakes. In: Proceedings of Europe's braking conference and exhibition, Dresden, Germany, 2015, p.18.

32. Perricone G, Mate jka V, Alemani M, et al. A concept for reducing PM 10 emissions for car brakes by 50\%. Wear 2018; 396-397: 135-145.

33. Nosko $\mathrm{O}$, Alemani $\mathrm{M}$ and Olofsson $\mathrm{U}$. Characterisation of airborne particles emitted from car brake materials. In: 6th World tribology congress, Beijing, China, 17-22 September 2017, p.5.

34. Ciudin R, Verma PC, Gialanella S, et al. Wear debris materials from brake systems: environmental and health issues. WIT Trans Ecol Environ 2014; 191: 1423-1434.

35. Alemani M, Perricone G, Olofsson U, et al. A proposed dyno bench test cycle to study particle emissions from disk brakes. In: Conference paper from Eurobrake 2014, Lille, France, 13-15 May 2014. London: FISITA.

36. Timte M. A comparison of lining output generated using AK master and FMVSS 105 simulation dynamometer procedures [Internet], https://www.sae.org/publications/ technicalpapers/content/2000-01-2777/ (2000, accessed 25 août 2020).

37. Gramstat S, Cserhati A, Schroeder M, et al. Brake particle emission measurements testing method and results. SAE Int J Engines 2017; 10(4): 1841-1846. 
38. Perricone G, Alemani M, Metino $\cdots z \mathrm{z}$, et al. Towards the ranking of airborne particle emissions from car brakes -a system approach. Proc IMechE, Part D: J Automobile Engineering 2017; 231(6): 781-797.

39. Demuynck J, Bosteels D, De Paepe M, et al. Recommendations for the new WLTP cycle based on an analysis of vehicle emission measurements on NEDC and CADC. Energy Policy 2012; 49: 234-242.

40. Kukutschova' J, Roubı'ček V, Malachova' K, et al. Wear mechanism in automotive brake materials, wear debris and its potential environmental impact. Wear 2009; 267(5-8): 807817.

41. lijima A, Sato K, Yano K, et al. Particle size and composition distribution analysis of automotive brake abrasion dusts for the evaluation of antimony sources of airborne particulate matter. Atmos Environ 2007; 41(23): 4908-4919.

42. Shin J, Yim I, Kwon S-B, et al. Evaluation of temperature effects on brake wear particles using clustered heatmaps. Environ Eng Res 2019; 24(4): 680-689.

43. O - sterle W, Bresch $\mathrm{H}$, Do * rfel I, et al. Surface film formation and dust generation during brake performance tests.

New York, NY: The Institution of Mechanical Engineers, 2009.

44. Alemani $M$, Wahlstro $\cdot m$ J, Mate jka $V$, et al. Scaling effects of measuring disc brake airborne particulate matter emissions - a comparison of a pin-on-disc tribometer and an inertia dynamometer bench under dragging conditions. Proc IMechE, Part $\mathrm{H}$ : J Engineering Tribology 2018; 232(12): 1538-1547.

45. Kwak J, Kim H, Lee J, et al. Characterization of nonexhaust coarse and fine particles from on-road driving and laboratory measurements. Sci Total Environ 2013; 458-460: 273-282.

46. Farwick zum Hagen FH, Mathissen M, Grabiec T, et al. On-road vehicle measurements of brake wear particle emissions. Atmos Environ 15 2019; 217: 116943.

47. Hagino $\mathrm{H}$. Feasible methodology for brake wear particles measurement and characterization. In: 45th PMP meeting, Joint Research Centre, Ispra, Italy, 7-8 November 2017, p.19.

48. Shabanian SR, Rahimi M, Shahhosseini M, et al. CFD and experimental studies on heat transfer enhancement in an air cooler equipped with different tube inserts. Int Commun Heat Mass Transfer 2011; 38(3): 383-390.

49. Agudelo C. PMP-45-15 LINK Design considerations for brake emission measurements. 2017.

50. Brembo S.p.A. REBRAKE: a brake test stand for particles measurement and collection. 2016.

51. Ansaloni S, Sin A and Moiso M. PMP-48-19 ITT presentation. 7-8 November 2018, Ispra, Italy.

52. Kulkarni P, Baron PA and Willeke K. Aerosol measurement: principles, techniques, and applications. Hoboken, NJ: John Wiley \& Sons, 2011, p.904.

53. Dennis R, Samples WR, Anderson DM, et al. Isokinetic sampling probes. Ind Eng Chem 1957; 49(2): 294-302.

54. Wilcox JD. Isokinetic flow and sampling. J Air Pollut Control Assoc 1956; 5(4): 226-245.

55. Breuer M, Baytekin HT and Matida EA. Prediction of aerosol deposition in 90 bends using LES and an efficient Lagrangian tracking method. J Aerosol Sci 2006; 37(11): 14071428. 
56. Sun K and Lu L. Particle flow behavior of distribution and deposition throughout 90 bends: analysis of influencing factors. J Aerosol Sci 2013; 65: 26-41.

57. Park SH, Kim HO, Han YT, et al. Wall loss rate of polydispersed aerosols. Aerosol Sci

Technol 2001; 35(3):

710-717.

58. Kim DS, Park SH, Song YM, et al. Brownian coagulation of polydisperse aerosols in the transition regime. J Aerosol Sci 2003; 34(7): 859-868. 14 Proc IMechE Part D: J Automobile Engineering 00(0)

59. Sherwood T and Wei J. Interfacial phenomena in liquid extraction. Ind Eng Chem 1957; 49(6): 1030-1034.

60. Sulaiman M, Climent E, Hammouti A, et al. Mass transfer towards a reactive particle in a fluid flow: numerical simulations and modeling. Chem Eng Sci 2019; 199: 496-507.

61. Delattre $P$ and Friedlander SK. Aerosol coagulation and diffusion in a turbulent jet. Ind Eng Chem Fund 1978; 17(3): 189-194.

62. Berrouk AS and Laurence D. Stochastic modelling of aerosol deposition for LES of 90 bend turbulent flow. Int J Heat Fluid Flow 2008; 29(4): 1010-1028.

63. Bluestein AM, Venters $R$, Bohl $D$, et al. Turbulent flow through a ducted elbow and plugged tee geometry: an experimental and numerical study. J Fluids Eng 2019; 141(8):

081101-081101-14.

64. Cheng YS and Wang CS. Motion of particles in bends of circular pipes. Atmos Environ 1981; 15(3): 301-306.

65. Cong XC, Yang GS, Qu JH, et al. A model for evaluating the particle penetration efficiency in a ninety-degree bend with a circular-cross section in laminar and turbulent flow regions. Powder Technol 2017; 305: 771-781.

66. Crane RI and Evans RI. Inertial deposition of particles in a bent pipe. J Aerosol Sci 1977; 8: 161-170.

67. Pui DYH, Romay-Novas F and Liu BYH. Experimental study of particle deposition in bends of circular cross section. Aerosol Sci Technol 1987; 7(3): 301-315.

68. Sun $\mathrm{K}$, Lu $\mathrm{L}$ and Jiang $\mathrm{H}$. A numerical study of bendinduced particle deposition in and behind duct bends. Build Environ 2012; 52: 77-87.

69. Tsai C-J and Pui DYH. Numerical study of particle deposition in bends of a circular crosssection-laminar

flow regime. Aerosol Sci Technol 1990; 12(4): 813-831.

70. Zhang P, Roberts RM and Be'nard A. Computational guidelines and an empirical model for particle deposition in curved pipes using an Eulerian-Lagrangian approach. J Aerosol Sci 2012; 53: 1-20.

71. Muyshondt A, McFarland AR and Anand NK. Deposition of aerosol particles in contraction fittings. Aerosol Sci Technol 1996; 24(3): 205-216.

72. von der Weiden S-L, Drewnick F and Borrmann S. Particle loss calculator - a new software tool for the assessment of the performance of aerosol inlet systems. Atmos Meas Tech 2009; 2(2): 479-494.

73. Chen $Q$ and Ahmadi G. Deposition of particles in a turbulent pipe flow. J Aerosol Sci 1997; 28(5): 789-796.

74. Pozorski J. Models of turbulent flows and particle dynamics. In: Minier J-P and Pozorski J (eds) Particles in

wall-bounded turbulent flows: deposition, re-suspension and agglomeration [Internet]. Cham: Springer International

Publishing, 2017, pp.97-150. 
75. Reynolds AM. A Lagrangian stochastic model for heavy particle deposition. J Colloid Interface Sci 1999; 15(1): 85-91.

76. Wood NB. A simple method for the calculation of turbulent deposition to smooth and rough surfaces. J Aerosol Sci 1981; 12(3): 275-290.

77. Bandyopadhyay PR and Balasubramanian R. Vortex Reynolds number in turbulent boundary layers. Theor Comput Fluid Dyn 1995; 7(2): 101-117.

78. HudmanMand Hugh MB. Large eddy simulation of turbulent pipe flow. In: Second international conference on CFD in the minerals and process industries, Melbourne, VIC, Australia, December 1999, pp.369-374. Melbourne: CSIRO Australia.

79. Liu $X$, Zhang $Y, W u Q$, et al. Study on the key structure parameters of a gravity settling chamber based on a flow field simulation. Eng Appl Comput Fluid Mech 2019; 13(1): 377395.

80. Hussein T, Hrus`ka A, Doha'nyosova' $P$, et al. Deposition rates on smooth surfaces and coagulation of aerosol particles inside a test chamber. Atmos Environ 2009; 43(4): 905-914. 81. Hussein T, Kubincova' L, Džumbova' L, et al. Deposition of aerosol particles on rough surfaces inside a test chamber. Build Environ 2009; 44(10): 2056-2063.

82. Zhao B and Wu J. Particle deposition in indoor environments: analysis of influencing factors. J Hazard Mater 2007; 147(1): 439-448.

83. Van Leussen W. Aggregation of particles, settling velocity of mud flocs a review. In: Dronkers $\mathrm{J}$ and van Leussen W (eds) Physical processes in estuaries. Berlin, Heidelberg: Springer, 1988, pp.347-403.

84. Keshani S, Daud WRW, Nourouzi MM, et al. Spray drying: an overview on wall deposition, process and modeling. J Food Eng 2015; 146: 152-162.

85. Lai ACK. Investigation of electrostatic forces on particle deposition in a test chamber. Indoor Built Environ 2006; 15(2): 179-186.

86. Stelling C, Mark A, Papastavrou G, et al. Showing particles their place: deterministic colloid immobilization by gold nanomeshes. Nanoscale 2016; 8(30): 14556-14564.

87. Yu K-P, Lee W-MG, Peng C-J, et al. Effects of roughness, dielectric constant and electrical resistivity of wall on deposition of submicron particles driven by ionic air purifier. $J$ Environ Chem Eng 2017; 5(4): 3108-3114.

88. Pratsinis SE and Kim K-S. Particle coagulation, diffusion and thermophoresis in laminar tube flows. J Aerosol Sci 1989; 20(1): 101-111.

89. Walsh JK,Weimer AW and Hrenya CM. Thermophoretic deposition of aerosol particles in laminar tube flow with mixed convection. J Aerosol Sci 2006; 37(6): 715-734.

90. Wahlstro - m J, Lyu Y, Matjeka V, et al. A pin-on-disc tribometer study of disc brake contact pairs with respect to wear and airborne particle emissions. Wear 2017; 384- 385: 124-130.

91. Wilson JC and Liu BYH. Aerodynamic particle size measurement by laser-Doppler velocimetry. J Aerosol Sci 1980; 11(2): 139-150.

92. Hergert $\mathrm{W}$ and Wriedt $\mathrm{T}$. The Mie theory: basics and applications. Berlin, Heidelberg: Springer, 2012, p.268.

93. Keskinen J, Pietarinen K and Lehtima * kiM. Electrical low pressure impactor. J Aerosol Sci 1992; 23(4): 353-360.

94. Marjama - ki M, Keskinen J, Chen D-R, et al. Performance evaluation of the electrical low-pressure impactor (ELPI). J Aerosol Sci 2000; 31(2): 249-261.

95. Wang SC and Flagan RC. Scanning electrical mobility spectrometer. Aerosol Sci Technol 1990; 13(2): 230-240. 
96. Price HD, Stahlmecke B, Arthur R, et al. Comparison of instruments for particle number size distribution measurements in air quality monitoring. J Aerosol Sci 2014; 76: 48-55.

97. Peters TM, Ott D and O'Shaughnessy PT. Comparison of the Grimm 1.108 and 1.109 portable aerosol spectrometer to the TSI 3321 aerodynamic particle sizer for dry particles. Ann Occup Hyg 2006; 50(8): 843-850. Philippe et al. 15

98. Tuch TH, Mirme A, Tamm E, et al. Comparison of two particle-size spectrometers for ambient aerosol measurements. Atmos Environ 2000; 34(1): 139-149.

99. Sto - ber W. A note on the aerodynamic diameter and the mobility of non-spherical aerosol particles. J Aerosol Sci 1971; 2(4): 453-456.

100. Thomas D, Charvet A, Bardin-Monnier N, et al. Aerosol filtration. Oxford: Elsevier, ISTE Press, 2016, p.228.

101. Sanders PG, Xu N, Dalka TM, et al. Airborne brake wear debris: size distributions, composition, and a comparison of dynamometer and vehicle tests. Environ Sci Technol 2003; 37(18): 4060-4069. 